\title{
Efeito do Nível de Saturação de Alumínio em Solo Ácido sobre os Danos de Spodoptera frugiperda (J. E. Smith) em Milho
}

Ivan Cruz ${ }^{1}$, Lenita J. Oliveira ${ }^{2}$, Antônio C. Oliveira ${ }^{1}$ e Carlos A. Vasconcelos ${ }^{1}$

${ }^{1}$ EMBRAPA/CNPMS, Caixa postal 151, 35701-970, Sete Lagoas, MG. ${ }^{2}$ EMBRAPA/CNPSo, Caixa postal 231, 86001-970, Londrina, PR.

An. Soc. Entomol. Brasil 25(2): 293-297 (1996)

\section{Effect of Aluminum Saturation Level in Acid Soil on Damage of Spodoptera} frugiperda (J. E. Smith) to Maize

\begin{abstract}
This study was conducted to verify the damage of artificial infestation of the fall armyworm, Spodoptera frugiperda (J. E. Smith) to maize cultivars at different levels of Al saturation (low, medium and high) in an acid soil. The experiments were conducted in the field, at Sete Lagoas, MG, during three consecutive years in plots corrected with lime to provide three levels of aluminum saturation: toxic $(\mathrm{m}<50 \%)$, intermediate $(\mathrm{m}<20 \%)$, and without aluminum $(\mathrm{m}<5 \%)$. Two maize cultivars, BR 136 (tolerant) and CMS 28 (sensitive) to toxic levels of Al saturation were used. The non-infested plots were maintained free from natural infestation of the fall armyworm using granular insecticide applied directly to the whorl. The treated plots were artificially infested with young larvae. The average yield losses due to fall armyworn were $848 \mathrm{~kg} / \mathrm{ha}(17.7 \%)$ and were constant across the treatments. The highest yield without infestation was $4792 \mathrm{~kg} / \mathrm{ha}$.
\end{abstract}

KEY WORDS: Insecta, soil fertility, yield losses, Zea maiz.

RESUMO - Estudou-se a influência de níveis de calagem na intensidade dos danos provocados pela lagarta-do-cartucho, Spodoptera frugiperda (J. E. Smith), em milho. Foram conduzidos experimentos durante três anos consecutivos, em parcelas que receberam calagem de modo a manter três níveis de saturação de alumínio: nível tóxico $(\mathrm{m}<50 \%)$, intermediário $(\mathrm{m}<20 \%)$ e sem alumínio $(\mathrm{m}<5 \%)$. Foram utilizadas duas cultivares de milho, a BR 136, tolerante à toxidez de alumínio, e a CMS 28, sensível. Foram também utilizadas unidades experimentais infestadas artificialmente com lagartas recém-nascidas, e não infestadas, controladas com inseticidas granulados aplicados diretamente no cartucho da planta. Independente da cultivar utilizada ou do nível de fertilidade do solo, as perdas quantitativas ocasionadas à cultura do milho foram de $17,7 \%$ ( $848 \mathrm{~kg} / \mathrm{ha})$, considerando uma produtividade máxima, sem o ataque da praga, de $4.792 \mathrm{~kg} / \mathrm{ha}$.

PALAVRAS-CHAVE: Insecta, fertilidade do solo, danos, Zea maiz.

Os danos ocasionados pela lagarta-docartucho do milho, Spodoptera frugiperda (J.
E. Smith), têm variado até um máximo de $38,7 \%$, dependendo da planta e de seu 
desenvolvimento fenológico (Carvalho 1970, Cruz \& Turpin 1982, 1983, Williams \& Davis 1990, Willink et al. 1991). Esses trabalhos foram realizados em condições de fertilidade apropriadas para o cultivo do milho. Estudos considerando variações na fertilidade do solo, especialmente em condições de baixa disponibilidade de nutrientes, são escassos. A biologia do inseto e aspectos de sua nutrição quando o milho foi cultivado em solo corrigido para diferentes níveis de alumínio foram estudados por Oliveira et al. (1987, 1990 a,b). Segundo Maxwell (1972) e Tandon (1973), trabalhos envolvendo níveis de fertilidade do solo em relação a insetos são complexos, pois podem ocorrer vários efeitos interativos nos diferentes estádios de desenvolvimento do inseto, além de aumentar a resistência das plantas à infestação da praga. No Brasil, Carvalho et al. (1984) estudaram o efeito de diferentes doses de potássio. Nessa mesma linha de pesquisa, são salientados, no exterior, os trabalhos de Wiseman et al. (1973 a,b), Leuck (1972), Leuck \& Hammons (1974), Leuck et al. (1974), Clavijo (1984) e Lynch (1984). Poucas são as informações obtidas para as diferentes pragas, para solos de cerrado, onde normalmente têm-se problemas para as plantas, em função do nível tóxico do alumínio, que interfere na disponibilidade dos nutrientes, à medida que cresce o pH (Bahia Filho et al. 1983). Neste trabalho, avaliou-se, a relação entre os danos causados por $S$. frugiperda em cultivares de milho, em solo de cerrado corrigido para três níveis de alumínio.

\section{Material e Métodos}

O trabalho foi realizado em Sete Lagoas, MG, no Centro Nacional de Pesquisa de Milho e Sorgo-CNPMS da EMBRAPA, por três anos consecutivos, em um Latossolo VermelhoEscuro, fase cerrado, com as seguintes características químicas iniciais: $\mathrm{pH}: 4,6, \mathrm{Al}$ : 1,3 meg/100 $\mathrm{cm}^{3}$, Ca: 0,9 meg/100 $\mathrm{cm}^{3}, \mathrm{Mg}$ : 0,2 meg/100 $\mathrm{cm}^{3}, \mathrm{~K}: 60 \mathrm{ppm}, \mathrm{P}: 13 \mathrm{ppm} \mathrm{e}$ matéria orgânica: 3,3\%, seguindo-se a metodologia descrita por Vettori (1969). O gradiente de saturação de alumínio no solo foi obtido utilizando-se duas doses de calcário dolomítico (2 e 6 t/ha), e uma testemunha sem calcário. Dessa maneira, foram obtidos os seguintes níveis de saturação de alumínio: $\mathrm{m}<$ $5 \%, \mathrm{~m}<20 \%$ e $\mathrm{m}<50 \%$. Periodicamente, foram feitas novas análises, e, quando necessário, principalmente para evitar "stress" acentuado, foram aplicadas quantidades pertinentes do mesmo tipo de calcário, procurando manter a saturação de alumínio. Em todos os experimentos, por ocasião do plantio, a cultura foi adubada com $400 \mathrm{~kg} / \mathrm{ha}$ da fórmula 4-14-8, adicionando-se também 5 $\mathrm{kg}$ de $\mathrm{Zn}$, e 40 dias após, sulfato de amônia em cobertura (200 kg/ha).

O delineamento experimental foi em blocos ao acaso, com parcelas subsubdivididas com três repetições. Os níveis de calcário, 0, 2 e 6 t/ha, foram dispostos nas parcelas. Os genótipos CMS 28, sensível a toxidez de alumínio, e o BR 136, tolerante, foram casualizados dentro de cada parcela, constituindo as subparcelas. Para cada genótipo, estabeleceram-se as subsubparcelas como níveis de infestação (com e sem). Os genótipos foram desenvolvidos na EMBRAPA/CNPMS, apresentando um potencial genético semelhante para produção de grãos, quando na ausência da toxidez de alumínio. As plantas não infestadas foram mantidas isentas de praga através de inseticidas granulados, aplicados dentro do cartucho. Cada planta infestada recebeu em média dez lagartas recém-nascidas, provenientes de criação artificial de laboratório, cerca de 40 dias após o plantio, por ser essa a época mais suscetível ao ataque da praga (Cruz \& Turpin 1982). Cada unidade experimental foi representada por 40 plantas. As avaliações basearam-se na produção de grãos de cada subparcela (padronizada para umidade de $15,5 \%$, obtida na colheita e analisada através da análise de variância. As médias foram separadas pelo teste de Duncan ao nível de $5 \%$ de significância. 


\section{Resultados e Discussão}

Análises químicas do solo efetuadas no início dos experimentos e após a colheita do último ano de pesquisa, respectivamente mostraram diferenças marcantes para os três níveis de calagem, principalmente no que se refere à saturação $\left(\mathrm{Al}^{3+} \mathrm{e}\right.$ teores de alumínio, $\mathrm{Ca}^{2+} \mathrm{e} \mathrm{Mg}^{2+}$. Os demais elementos permaneceram constantes (Tabela 1). efeito significativo em todos os níveis de calagem, indepen- dentemente do ano e do genótipo (Tabela 2). Pode-se verificar que, na ausência de cala- gem, quando as condições do solo apre- sentavam-se tóxicas para a planta, em termos de alumínio, a queda na produtividade de grãos, em média, foi de 515 $\mathrm{kg} / \mathrm{ha}$, enquanto que nas condições ideais $(6 \mathrm{t} /$ ha de calcário) foi de $1.037 \mathrm{~kg} / \mathrm{ha}$, ou seja, a queda no rendimento foi mais pronunciada em

Tabela 1. Análise química de solo sujeito a diferentes doses de calcário dolomítico, efetuada em duas épocas, início e final dos experimentos após três anos.

\begin{tabular}{|c|c|c|c|c|c|c|c|c|c|}
\hline \multirow[t]{3}{*}{ Época } & \multirow{3}{*}{$\begin{array}{l}\text { Doses de } \\
\text { calcário } \\
\text { (t/ha) }\end{array}$} & \multicolumn{8}{|c|}{ Resultados analíticos } \\
\hline & & \multirow[t]{2}{*}{$\mathrm{pH}$} & $\mathrm{Al}$ & $\mathrm{Ca}$ & $\mathrm{Mg}$ & $\mathrm{K}$ & $\mathrm{P}$ & \multirow{2}{*}{$\frac{\text { Mo }}{(\%)}$} & \multirow{2}{*}{$\begin{array}{l}\text { Saturação } \\
\text { de } \mathrm{Al}(\%)\end{array}$} \\
\hline & & & \multicolumn{3}{|c|}{ eq.mg/100 cc } & \multicolumn{2}{|c|}{ ppm } & & \\
\hline \multirow[t]{3}{*}{ Início } & 0 & 4,6 & 1,70 & 1,08 & 0,19 & 59 & 13 & 3,31 & 54 \\
\hline & 2 & 4,9 & 1,00 & 2,06 & 0,67 & 68 & 17 & 3,44 & 26 \\
\hline & 6 & 5,3 & 0,20 & 3,10 & 1,28 & 65 & 13 & 3,39 & 4 \\
\hline \multirow[t]{3}{*}{ Final } & 0 & 4,8 & 1,63 & 0,74 & 0,15 & 64 & 8 & 3,17 & 61 \\
\hline & 2 & 5,1 & 1,00 & 1,40 & 0,46 & 58 & 9 & 3,49 & 33 \\
\hline & 6 & 5,4 & 0,45 & 2,29 & 0,88 & 50 & 8 & 3,52 & 12 \\
\hline
\end{tabular}

O genótipo BR 136, tolerante à acidez do solo, apresentou-se significativamente mais produtivo do que o genótipo sensível CMS 28 , na ausência da calagem. Na média de três anos de pesquisa e independente da infestação, houve uma diferença de produtividade de $1.090 \mathrm{~kg} / \mathrm{ha}$, em favor do BR 136 , o que representa aproximadamente $44,0 \%$. Nos níveis de calagem de 2 t/ha e 6 t/ha, as diferenças observadas nas produtividades dos dois genótipos, $213 \mathrm{~kg} / \mathrm{ha}$ e $241 \mathrm{~kg} / \mathrm{ha}(4,7$ $\%)$, respectivamente, não foram significativas, ao nível de $5 \%$ de probabilidade pela análise de variância (Tabela 2).

Nos três anos de pesquisa os resultados mostraram, de forma consistente, o efeito significativo do dano provocado por $S$. frugiperda sobre a produtividade de milho. A infestação, que mede o referido dano, apresentou-se com condições de maior produtividade. Convém ressaltar, no entanto, que o percentual de perda de grãos devido à praga (efeito da infestação) permanece constante, em torno de 17,7\% (Tabela 2). Os dados obtidos neste trabalho são semelhantes aos obtidos por Cruz \& Turpin (1982), que também trabalharam com infestações artificiais. Esses autores encontraram perdas médias de $18,7 \%$ em dois anos de estudos. Carvalho (1970), trabalhando com infestações naturais, encontrou perdas bem maiores, chegando a um máximo de $34 \%$, quando o ataque ocorreu na época de florescimento. Essas perdas foram de 15,4 e $29,7 \%$, respectivamente, para ataques na época do desbaste ou aos 49 dias após o plantio. Em função dos dados obtidos, pode-se concluir que, independentemente da cultura utilizada (sensível ou tolerante ao alumínio) ou da 
Tabela 2. Produção (média \pm EP) de grãos ( $\mathrm{kg} / \mathrm{ha}$ ) de duas cultivares de milho, submetidas à infestação artificial por Spodoptera frugiperda em três níveis de calagem e três anos agrícolas.

\begin{tabular}{|c|c|c|c|c|c|}
\hline \multirow{2}{*}{$\begin{array}{l}\text { Calagem } \\
\text { (t/ha) }\end{array}$} & \multirow[t]{2}{*}{ Infestação } & \multicolumn{3}{|c|}{ Produtividade de três anos por cultivar $(\mathrm{kg} / \mathrm{ha})$} & \multirow{2}{*}{$\begin{array}{c}\text { Valor } \\
\text { percentual }\end{array}$} \\
\hline & & BR 136 & CMS 28 & Média geral ${ }^{1}$ & \\
\hline \multirow[t]{2}{*}{0} & sem & $3986 \pm 268$ & $2612 \pm 158$ & 3299 a & 100 \\
\hline & com & $3187 \pm 496$ & $2381 \pm 238$ & $2784 \mathrm{~b}$ & 84 \\
\hline Média & & 3586 & 2496 & 3041 & \\
\hline \multirow[t]{2}{*}{2} & sem & $5348 \pm 381$ & $5293 \pm 85$ & 5320 a & 100 \\
\hline & com & $4518 \pm 339$ & $4148 \pm 75$ & $4333 \mathrm{~b}$ & 81 \\
\hline Média & & 4933 & 4720 & 4826 & \\
\hline \multirow[t]{2}{*}{6} & sem & $5820 \pm 244$ & $5682 \pm 292$ & 5751 a & 100 \\
\hline & com & $4846 \pm 291$ & $4543 \pm 195$ & $4714 b$ & 82 \\
\hline Média & & 5353 & 5112 & 5232 & \\
\hline \multicolumn{2}{|c|}{ Média geral } & 4624 & 4109 & & \\
\hline
\end{tabular}

${ }^{1}$ Médias seguidas por letras diferentes, na coluna, para uma mesma dose de calcário, diferem significativamente entre si ao nível de $5 \%$ (Duncan).

disponibilidade de nutrientes para a planta, em função da presença ou ausência de alumínio no solo, as perdas na produção de grãos na cultura do milho, ocasionadas pela $S$. frugiperda, foram em torno de $18 \%$. Portanto, quanto maior a produtividade esperada, maior retorno poderá ser esperado com a adoção de medidas de controle da praga na cultura de milho.

\section{Literatura Citada}

Bahia Filho, A.F.C., C.A.Vasconcelos, H.L. Santos, G.E. França \& G.V.E. Pitta. 1983. Nutrição e adubação do milho. EMBRAPA/CNPMS, Documentos, 3, $44 \mathrm{p}$.

Carvalho, R.P.L. 1970. Danos, flutuação de população, controle e comportamento de Spodoptera frugiperda (J.E. Smith, 1794) susceptibilidade de diferentes genótipos de milho em condições de campo. Tese doutorado, ESALQ/USP, Piracicaba, 170 p.
Carvalho, R.B., M.M. Tristão, E. Giacon, M.H. Calafiori, N.T. Teixeira \& B.F. Bueno. 1984. Estudo de diferentes dosagens de potássio em milho (Zea mays L.) influindo sobre Spodoptera frugi- perda (J.E. Smith, 1797). Ecossistema 9: 95-100.

Clavijo, S. 1984. Effects of nitrogen fertilization and different levels of infes- tation by Spodoptera frugiperda (Lepi- doptera: Noctuidae) on the yields of maize. Rev. Fac. Agron., Maracay, 13: 43-48.

Cruz, I. \& F.T. Turpin. 1982. Efeito da Spodoptera frugiperda em diferentes estágios de crescimento da cultura de milho. Pesq. Agropec. Bras. 17: 355-359.

Cruz, I. \& F. T. Turpin. 1983. Yield impact of larval infestation of the fall armyworm Spodoptera frugiperda (J.E. Smith) to mid-whorl growth stage of corn. J. Econ. Entomol. 76:1052-1054. 
Leuck, D.B. 1972. Induced fall armyworm resistance in pearl millet. J. Econ. Entomol. 5:1608-1611.

Leuck, D.B. \& R.O. Hammons. 1974. Nutrients and growth media: influence on expression to resistance to the fall armyworm in the peanut. J. Econ. Entomol. 67: 564 .

Leuck, D.B., B.R. Wiseman \& W.W. McMillian. 1974. Nutritional plant sprays: effect on fall armyworm feeding preferences. J. Econ. Entomol. 67: 58-60.

Lynch, R.E. 1984. Effect of Coastal Bermudagrass fertilization levels and age of regrowth on fall armyworm (Lepidoptera: Noctuidae): larval biology and adult fecundity. J. Econ. Entomol. 77: 948-953.

Maxwell, F.G. 1972. Host plant resistance to insects - nutritional and pest management relationships, p. 599-609. In J.G. Ro-drigues (ed.), Insect and mite nutrition. Netherlands, North Holland Publ. Comp. 702p.

Oliveira, L.J. 1987. Biologia, nutrição quantitativa e danos causados por Spodoptera frugiperda em milho cultivado em solo corrigido para três níveis de alumínio. Piracicaba, Dissertação mestrado, ESALQ/USP, Piracicaba, $125 \mathrm{p}$.

Oliveira, L.J., J.R.P. Parra \& I. Cruz. 1990a. Biologia da lagarta do cartucho em milho cultivado em solo corrigido para três níveis de alumínio. Pesq. Agropec. Bras. 25: 157-166.

Oliveira, L.J., J.R.P. Parra, \& I. Cruz. 1990b. Nutrição quantitativa de lagarta do cartucho em milho cultivado para três níveis de alumínio. Pesq. Agropec. Bras. 25: $235-241$.

Tandon, H.L.S. 1973. The crop nutritionpest incidence complex in India. PANS 19: $372-380$.

Vettori, L. 1969. Métodos de análise de solo. Bol. Téc. EPE. 7: 1-24.

Williams, W.P. \& F.M. Davis. 1990. Response of corn to artificial infestation with fall armyworm and southwestern corn borer larvae. Sothwest. Entomol. 15: 163-166.

Willink, E., V.M. Osores \& M.A. Costilla. 1991. El gusano "cogollero" del maiz. Avance Agroind. 7: 3-7

Wiseman, B.R., D.B. Leuck \& W.W. McMillian. 1973a. Effect of crop fertilizer on feeding of larvae of fall armyworm on excised leaf sections of corn foliage. J. Ga. Entomol. Soc. 8: 136-141.

Wiseman, B.R., D.B. Leuck \& W.W. McMillian. 1973b. Increasing susceptibility and resistance of an inter-mediate resistant Antiqua corn to fall armyworm and corn earworm larvae by fertilizer treatments. Fla. Entomol. 56:1-7.

Recebido em 06/06/95. Aceito em 13/06/96. 